\title{
Single-cell genomics to understand disease pathogenesis
}

\author{
Seitaro Nomura ${ }^{1}$
}

Received: 1 July 2020 / Revised: 1 September 2020 / Accepted: 1 September 2020 / Published online: 19 September 2020

(c) The Author(s) 2020. This article is published with open access

\begin{abstract}
Cells are minimal functional units in biological phenomena, and therefore single-cell analysis is needed to understand the molecular behavior leading to cellular function in organisms. In addition, omics analysis technology can be used to identify essential molecular mechanisms in an unbiased manner. Recently, single-cell genomics has unveiled hidden molecular systems leading to disease pathogenesis in patients. In this review, I summarize the recent advances in single-cell genomics for the understanding of disease pathogenesis and discuss future perspectives.
\end{abstract}

\section{Single-cell genomics to dissect the biology of heart failure}

The heart constantly responds to hemodynamic overload. Cardiomyocytes, which are the principal components of the pump function of the heart, are required to maintain cardiac homeostasis by adapting appropriately to this stress. However, sustained exposure to pathological stress disrupts the adaptive mechanisms of cardiomyocytes, leading to heart failure. Understanding how each cardiomyocyte responds to various stimuli at the single-cell level will help to elucidate the pathogenesis of heart failure.

Cardiomyocytes are cylindrically shaped, around $120-\mu \mathrm{m}$ long and $30-\mu \mathrm{m}$ wide. Because of this large size, See et al. decided to isolate nuclei from cardiomyocytes using a microfluidics system and conducted single-nucleus RNAsequencing (RNA-seq) to reveal the activation of cell-cycle regulators and novel long noncoding RNAs in diseased cardiomyocytes [1]. To obtain expression profiles from single cardiomyocytes, we modified the Smart-seq2 protocol $[2,3]$, which amplifies full-length cDNA for single-cell transcriptome analysis and established a system to quantitatively analyze the transcriptomes of manually picked live single cardiomyocytes [4].

We applied this system to a mouse model of pressure overload-induced heart failure and obtained single-cell

Seitaro Nomura

senomura-cib@umin.ac.jp

1 Department of Cardiovascular Medicine, Graduate School of Medicine, University of Tokyo, Tokyo, Japan transcriptomes of cardiomyocytes isolated during the progression of heart failure [4]. Weighted gene co-expression network analysis, which extracts gene modules coexpressed across cells [5], identified nine gene modules and, using the expression profiles of these gene modules, we classified cardiomyocytes into seven cell states. Pseudotime analysis with the machine learning algorithm Monocle [6] identified two distinct trajectories for adaptive and failing cardiomyocytes. Chromatin immunoprecipitation using an anti-H3K27ac antibody followed by sequencing revealed the regulatory elements of the gene modules and inferred the upstream transcription factors associated with cardiomyocyte hypertrophy and failure. Through these analyses, we revealed that DNA damage and p53 signaling are activated at the branch point for failing cardiomyocytes, and this enabled us to generate cardiomyocyte-specific p53 knockout mice and show that p53 is essential for the induction of failing cardiomyocytes.

To recover the spatial information lost in single-cell RNA-seq analysis, we established a single-molecule RNA in situ hybridization assay, which enables quantification of each mRNA at the single-cell level [7], and revealed the spatial heterogeneity of failing cardiomyocytes induced by pressure overload [8]. Furthermore, the single-cell RNA-seq profiles of cardiomyocytes isolated from patients with heart failure also validated the presence of failing cardiomyocytes, characterized by the activation of DNA damage response genes, only in patients showing poor prognosis [4]. We also performed molecular pathology analysis using cardiac biopsy samples from patients with heart failure before treatment to demonstrate that the level of DNA damage in cardiomyocytes determines clinical prognosis and treatment response [9]. Recent single-cell RNA-seq 


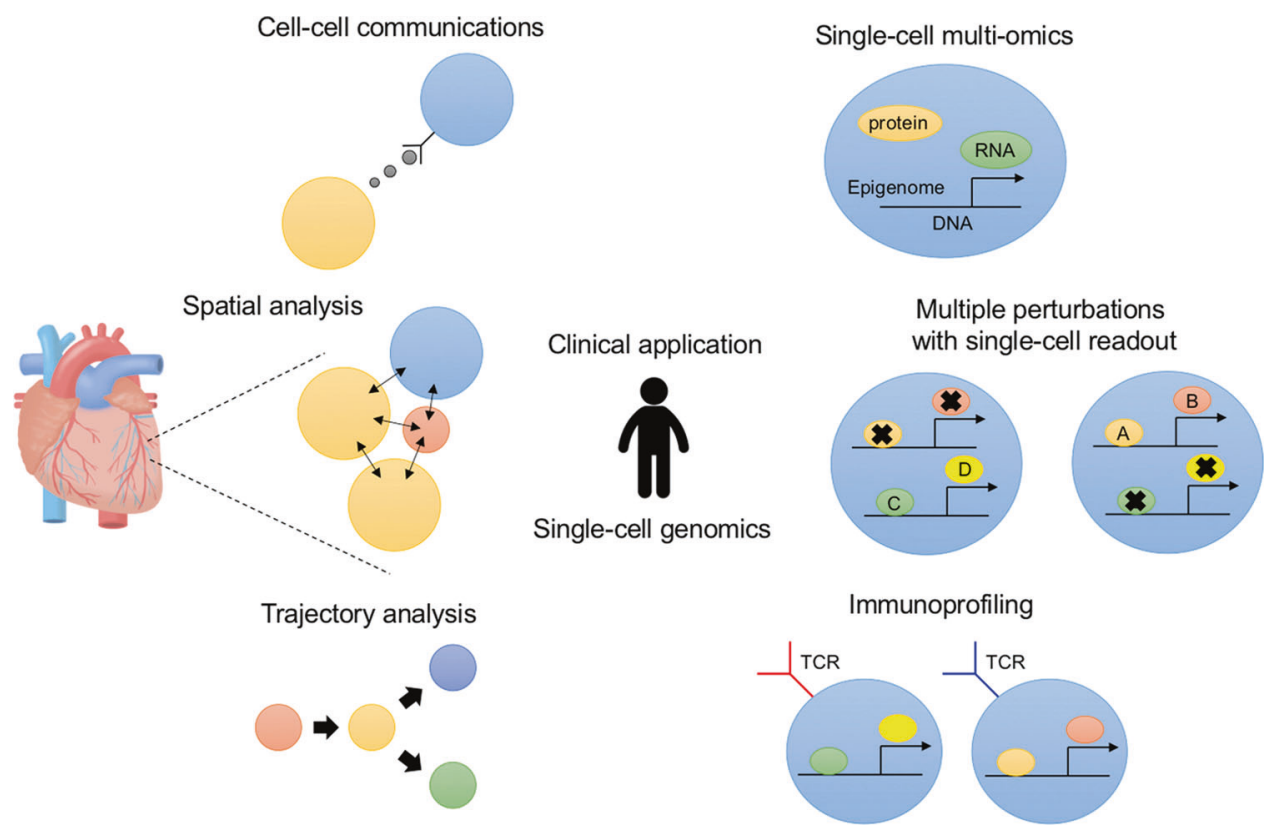

Fig. 1 Overview of single-cell genomics to understand disease pathogenesis.

analysis in heart failure biology has also revealed the involvement of inflammatory cells [10] and the anatomical expression profiles of disease-causing genes [11, 12].

We and others have applied single-cell genomics and used cell-type classification, trajectory inference, marker identification, spatial analysis, and clinical assessment to further our understanding of disease pathogenesis. However, conventional single-cell RNA-seq analysis is not sufficient for obtaining the information necessary for a deeper understanding of molecular behavior. Recently, several studies have opened new avenues by focusing on cell-cell communications, spatial single-cell omics, barcode lineage tracing, single-cell multi-omics, multiple perturbations with single-cell readout, immunoprofiling, and clinical application (Fig. 1). In this review, I discuss the recent advances and future perspectives of single-cell genomics in our efforts to understand development, physiology, and pathophysiology.

\section{Cell-cell communications}

Several types of cells interact with each other via a variety of signaling molecules to generate organ-level functions. By integrating the single-cell expression profiles of ligands and receptors in developing lung tissue with a ligand and receptor interaction database, Cohen et al. generated a cell-cell communication map and identified eosinophils as essential signal mediators in the lung [13]. Eosinophils express IL1RL1, which binds to IL-33 secreted from alveolar epithelial type II cells, and secrete IL-6 or IL-13 to activate macrophages and maintain the immune environment in the lung. Vento-Tormo et al. leveraged the expression profiles of $\sim 70,000$ single cells from firsttrimester placentas with matched maternal blood and decidual cells, revealing the cellular organization of the decidua and placenta and the interactions that are critical for placentation and reproductive success [14]. They identified three types of decidual natural killer (dNK) cells: dNK1 cells secrete CSF1 to transduce signals to extravillous trophoblasts and macrophages; dNK2 cells secrete XCL1 to transduce signals to extravillous trophoblasts and dendritic cells; and dNK3 cells secrete CCL5 to transduce signals to extravillous trophoblasts and macrophages. They also developed CellPhoneDB, an algorithm implemented by Python, to enable cell-cell communication analysis that considers the structural composition of ligands and receptors [15].

Giladi et al. reported an approach for sequencing physically interacting cells (PIC-seq), which integrates cell sorting of physically interacting cells (PICs) with single-cell RNA-seq data, to comprehensively investigate the functional nature of PICs and identify the signaling molecules associated with these interactions [16]. In the developing lung, PICs of regulatory $\mathrm{T}$ cells and dendritic cells specifically express IL12b, whereas PICs of regulatory $\mathrm{T}$ cells and monocytes express CCL6. However, this method needs detailed consideration of the conditions used and validation of the identified signaling molecules by alternative approaches.

Cell-cell communications also occur at the level of the organism. Ma et al. obtained $\sim 210,000$ single-cell transcriptomes from several organs (adipose tissue, aorta, kidney, liver, skin, bone marrow, brain, and skeletal muscle) 
from young and aged rats with or without calorie restriction and analyzed the changes in cellular distribution and celltype-specific expression profiles [17]. They revealed an increase of cell types associated with immunity and inflammation during aging across whole organs, which was alleviated by calorie restriction. Using SCENIC, an algorithm for inferring transcriptional networks [18], they also identified a decrease in the activity of specific transcription factors such as Cebpd and Cebpb during aging, which was also alleviated by calorie restriction. Cell-cell communication analysis uncovered an increase in the interactions associated with endothelial cells during aging, which was also rescued by calorie restriction.

\section{Spatial analysis}

Cell-cell communications can be assessed more accurately by in situ analysis with preservation of spatial information in tissues. Individual mRNA molecules can be detected accurately in cells with single-molecule fluorescence in situ hybridization (FISH) [19]. Eng et al. developed sequential FISH and quantitatively analyzed the mRNAs of 10,000 genes in the cortex, subventricular zone, and olfactory bulb of the mouse brain at the single-cell level [20]. This method not only allows unbiased identification of cell classes and their spatial organization but also reveals subcellular mRNA localization patterns and ligand receptor pairs across neighboring cells. By using the FISH method, $\mathrm{Su}$ et al. demonstrated simultaneous imaging of more than 1000 genomic loci and nascent transcripts of more than 1000 genes together with landmark nuclear structures, revealing that transcription activity correlates with the local enrichment of active chromatin, which consists of long-range chromatin interactions [21].

Multiplexed proteome approaches also enable quantitative analysis of protein expression levels in situ at the single-cell level [22]. Goltsev et al. developed a highly multiplexed cytometric imaging approach, termed codetection by indexing (CODEX), in which all target proteins are labeled simultaneously using DNA-conjugated antibodies and antibody identity is revealed by iterative exchange of fluorophore-conjugated DNA [23]. By analyzing the effect of the cellular neighborhood on the expression of receptors in splenic immune cells, they revealed the emergence of erythroblasts and disease-specific regulatory $\mathrm{T}$ cells and identified their interactions with dendritic cells. Schürch et al. re-engineered the CODEX method to be compatible with formalin-fixed paraffinembedded tissue, conducted simultaneous profiling using 56 protein markers in 140 tissue regions from 35 patients with advanced-stage colorectal cancer, and identified 9 conserved, distinct cellular neighborhoods, which are a collection of components characteristic of the immune tumor microenvironment in colorectal cancer [24]. Jackson et al. used mass cytometry imaging analysis, which allows labeling of all target proteins with heavy-metal-conjugated antibodies and quantification by point-by-point ablation of the samples coupled to mass spectrometry, and simultaneously quantified 35 biomarkers in $\sim 170,000$ cells on tissue specimens from nearly 350 patients with breast cancer [25]. Spatial single-cell analysis identified the phenotypes of tumor and stromal cells and their interaction patterns; using them, they stratified the patients into 20 subtypes, which were critically associated with clinical course.

Rodriques et al. developed Slide-seq, a method for transferring tissue sections onto a surface covered with DNA-barcoded beads in known positions [26]. Using this method, they revealed the spatial localization of several types of cells in the cerebellum and hippocampus and celltype-specific spatial responses in the cerebral cortex. At $3 \mathrm{~h}$ after injury, early response genes such as Fos and ribosomal RNAs are activated at the injured position; at 3 days after injury, cell-cycle-related genes are activated in microglia and macrophages in remote regions; at 2 weeks after injury, these cells are replaced to fill the injured position. These cells also showed the activation of genes involved in the development of oligodendrocytes.

Lundeberg et al. developed spatial transcriptomics, an approach similar to Slide-seq [27], and performed spatial gene expression analysis of human heart development [28]. This method is now accessible as Visium from $10 \times$ Genomics. They revealed the distinct behavior of cardiac neural crest cells, marked by ISL1 and STMN2, and Schwann cell precursors, marked by $A L D H 1 A 1$. Both cells emerge in the outflow tract, but only the latter localizes in the subepicardial interstitium during heart development. Even in the outflow tract, the former emerges only in the early phase, whereas the latter is found only in the late phase.

Recently, a variety of tissue clearing methods have been developed that can be applied to single-cell protein expression analysis in several organs [29]. Although most protein expression analyses are based on immunostaining, clearing-enhanced 3D imaging combines tissue clearing with single-molecule RNA in situ hybridization [7] to enable RNA localization analysis in transparent organs [30]. These tissue clearing methods need careful consideration, such as how the clearing reagents should be selected and whether the antibodies or probes are well distributed throughout the tissue.

\section{Trajectory analysis}

Trapnell et al. developed Monocle, an algorithm-based application for pseudotime analysis and inference of the linear or bifurcating trajectories of an individual cell's progress through differentiation $[6,31]$; to the current 
version is Monocle 3 [32, 33]. The algorithm reduces dimensions by using uniform manifold approximation and projection (UMAP) [34], clusters neighboring cells into groups with the Leiden method, and extracts the trajectories connecting groups. They applied this algorithm to singlecell transcriptomes of over 200,000 cells isolated from developing mouse embryos and identified more than 500 cell types and 56 developmental trajectories [32]. They also used the single-cell expression profiles of 86,000 cells isolated from developing Caenorhabditis elegans and identified 502 cell types and 1068 developmental trajectories [33]. They further showed that the integration of UMAP and Louvain clustering enables the identification of gene groups that correspond to protein complexes and pathways [35].

Additional information, such as the ratio of reads mapped to introns and exons, RNA metabolism, and protein expression profiles, enables trajectories to be inferred more accurately. On the basis of the concept that transcriptionally active cells have more unspliced mRNAs, La Manno et al. developed Velocyto, an algorithm for inferring trajectories using the ratio of reads mapped to introns and exons [36]. They applied this algorithm to single-cell RNA-seq data from the mouse hippocampus and identified several trajectories from neuroblasts to the subiculum and astrocytes. They also revealed the kinetics of transcription during human embryonic glutamatergic neurogenesis.

Because Monocle and Velocyto infer cellular trajectories by using single-cell information derived only from RNA molecules, these algorithms cannot accurately reconstruct trajectories in cell-state transitions such as endothelial-mesenchymal transition. Recently, on the basis of the concept that daughter cells generally have the same genome, lineage tracing analysis using DNA barcode technology, a method of lineage identification that uses a short section of re-writable DNA, has been advancing [37]. Approaches for generating DNA barcodes include retrovirus-induced genome insertion [38, 39], Cre/loxPmediated recombination [40-42], and CRISPR/Cas9-mediated DNA double-strand breaks [43-46]. Several approaches can read out barcode information as mRNA molecules, enabling the simultaneous detection of gene expression and lineage information $[42,45,46]$. Alemany et al. performed the simultaneous analysis of gene expression and lineage tracing in zebrafish and revealed that epidermal and mesenchymal cells in the caudal fin arise from the same progenitors and that osteoblast-restricted precursors can produce mesenchymal cells during regeneration after injury $[45,47]$. They also identified resident immune cells in the fin with a distinct clonal origin from other blood cell types. Bowling et al. established the CRISPR array repair lineage tracing mouse line and uncovered a clonal bottleneck in the response of hematopoietic stem cells to injury [46]. Pei et al. developed the PolyloxExpress mouse line, which shows Cre recombinase-dependent DNA barcoding that allows the parallel readout of barcodes and transcriptomes in single cells, revealed the molecular signature of differentiationinactive hematopoietic stem cells, and demonstrated that these cells can undergo symmetric self-renewal [42]. Frieda et al. established a synthetic system, termed memory by engineered mutagenesis with optical in situ readout, which is based on a set of barcoded recording elements (scratchpad). The scratchpad altered by CRISPR/Cas9based mutagenesis can be read out through multiplexed single-molecule RNA FISH, enabling the simultaneous detection of lineages and gene expression profiles in situ [48].

\section{Single-cell multi-omics analysis}

The simultaneous detection of DNA sequences and RNA expression profiles enables the identification of diseasecausing variants and their association with gene expression. There are novel methods to simultaneously extract information from DNA and RNA [49-51]. By physically separating mRNA from genomic DNA using oligo-dT bead capture and performing whole-transcriptome and wholegenome amplifications, Macaulay et al. developed a method that can detect thousands of transcripts in parallel with the genetic variants captured by DNA-seq data from single cells $[49,50]$. Dey et al. reported a quasilinear amplification strategy to quantify genomic DNA and mRNA from single cells without physical separation and showed that genes with high cell-to-cell variability in transcript numbers generally have lower genomic copy numbers, suggesting that copy number variation may drive variability in gene expression among individual cells [51].

The detection of single nucleotide variants using abundant single-cell RNA-seq data is an applicable and costeffective method for identifying expressed variants, inferring sub-clones, and deciphering genotype-phenotype relationships [52]. Enge et al. simultaneously analyzed single nucleotide variants and gene expression profiles from 2544 pancreatic cells from 8 donors and found that islet endocrine cells from older donors show increased levels of transcriptional noise and potential fate drift, which was considered to be induced by oxidative stress. By determining the mutational history of individual cells, they revealed a novel mutational signature in healthy aging endocrine cells [53]. Nam et al. developed genotyping of transcriptomes, a method to integrate genotyping with droplet-based single-cell transcriptomes, and used it to profile $~ 40,000 \mathrm{CD} 34+$ cells from patients with CALRmutated myeloproliferative neoplasms, identifying an association between the activation of the unfolded protein response and NF-kB pathway with CALR mutations [54]. 
Given that gene expression is regulated by the epigenome, simultaneous analysis of the transcriptome and epigenome leads to a deeper understanding of gene regulation. There are methods to simultaneously detect combinations of RNA expression and DNA methylation [55], chromatin accessibility, DNA methylation, and RNA expression [56-58], chromatin accessibility and RNA expression [59-61], protein-DNA interactions and RNA expression $[62,63]$, and high-order chromatin structure and RNA expression [64]. Argelaguet et al. performed single-cell nucleosome, methylation, and transcriptome sequencing of 1105 cells from the onset of gastrulation in mouse embryos [58]. Cells committed to the mesoderm and endoderm undergo widespread coordinated epigenetic rearrangements at enhancers, which are driven by 10-11 translocationmediated demethylation and an accompanying increase of chromatin accessibility. By contrast, the DNA methylation and chromatin accessibility landscape of ectodermal cells is already established in the early epiblast. Mateo et al. established optical reconstruction of chromatin architecture (ORCA), a method that can accurately detect the positions of DNA and RNA using array-derived oligonucleotide probes in the nucleus [64]. ORCA analysis of Drosophila embryos identified cell-type-specific physical borders between active and Polycomb-repressed DNA, and Polycomb-independent borders. Deletion of the Polycombindependent borders leads to ectopic contacts between enhancers and promoters, resulting in aberrant gene expression and developmental defects. Katzenelenbogen et al. developed intracellular staining and sequencing (i.e., INs-seq) that enables simultaneous detection of the intracellular signaling and protein state as well as the cellular transcriptional profiles, and identified $\operatorname{Arg} 1^{+}{ }^{+} \mathrm{Trem}^{+}$regulatory myeloid cells, which control tumor growth [65].

\section{Multiple perturbations with single-cell readout}

A combination of CRISPR/Cas9-based genetic screening and single-cell omics analysis enables comprehensive and detailed functional analyses [66-70]. Norman et al. integrated not only CRISPR interference (CRISPRi) but also CRISPR activation (CRISPRa) with single-cell RNA-seq to present an analytical framework for interpreting high-dimensional landscapes of cell states, and enabled the ordering of regulatory pathways, classification of genetic interactions, and mechanistic elucidation of synergistic interactions, including the cooperative function of $C B L$ and $C N N 1$ for driving erythroid differentiation [71]. By titrating expression using CRISPRi and a series of single-guide RNAs (sgRNAs) in human myeloid leukemia K562 cells, Jost et al. showed that a reduction in the mRNA levels of HSPA5 and GATAl by $\sim 50 \%$ is sufficient to induce a near maximal transcriptional response and growth defect, whereas a larger reduction of other genes is required for a similar effect, suggesting the sharp transition in cellular behavior at gene-specific expression thresholds [72]. Replogle et al. reported direct capture Perturb-seq, a method in which expressed sgRNAs are sequenced together with single-cell transcriptomes, and allowed pooled single-cell CRISPR screens to be paired easily with combinatorial perturbation libraries, improving the efficacy of CRISPRi and CRISPRa [73].

Multiple perturbations with single-cell readout has been developed to analyze epigenetic regulation [74], enhancer-promoter interactions [75], protein expression [76], and morphological and phenotypical assessments [77]. Rubin et al. developed the Perturb-assay for transposaseaccessible chromatin (ATAC) to detect gRNA information and chromatin accessibility simultaneously, using it to assess the synergistic effects of various transcription factors and epigenomic regulators on epigenomic regulation [74]. Gasperini et al. generated a gRNA library targeting 5920 enhancer regions in K562 cells, performed CRISPR/Cas9mediated perturbations followed by single-cell RNA-seq, and identified 664 enhancer-promoter interaction pairs [75]. By using targeted in situ sequencing of perturbations, Feldman et al. integrated CRISPRi with optical assessment [77]. By screening a set of 952 genes for involvement in NF- $\mathrm{KB}$ signaling by imaging the nuclear translocation of RelA (p65), they identified the importance of Mediator complex subunits such as MED12 and MED24 in regulation of the duration of p65 nuclear retention.

\section{Immunoprofiling}

The diversity of the vertebrate adaptive immune system is based on somatic rearrangements of $\mathrm{V}(\mathrm{D}) \mathrm{J}$ genes encoding the T-cell receptor (TCR) $\alpha$ and $\beta$ chains; therefore, simultaneous analysis of TCR sequence (clonality) and gene expression from individual cells provides a deeper understanding of molecular behavior in the adaptive immune system. By integrating single-cell transcriptomes with clonal information during the development of the human thymus, Park et al. identified a strong bias in V(D)J usage shaped by recombination and multiple rounds of selection, including a TCR $\alpha$ V-J bias for $\mathrm{CD} 8^{+} \mathrm{T}$ cells [78] Through performing single-cell RNA and TCR sequencing of tumor and normal tissues and peripheral blood in patients with different types of cancer, $\mathrm{Wu}$ et al. found that patients who show clonal expansion of effector-like $\mathrm{T}$ cells in tumor tissue as well as in peripheral blood respond well to antiPDL1 therapy [79]. Gate et al. integrated single-cell RNAseq with TCR-seq of peripheral blood mononuclear cells and cerebrospinal fluid from patients with Alzheimer's disease and identified an association between clonally 
expanded $\mathrm{CD}^{+}{ }^{+} \mathrm{T}$ effector memory $\mathrm{CD} 45 \mathrm{RA}^{+}$cells and disease severity [80]. The machine learning algorithm grouping of lymphocyte interactions by paratope hotspots [81] and cloning and peptide screens demonstrated the specificity of clonally expanded TCRs to two separate Epstein-Barr virus antigens. Oh et al. conducted single-cell RNA and paired TCR sequencing of 30,604 T cells from seven patients with bladder cancer and found multiple cytotoxic $\mathrm{CD} 4+\mathrm{T}$ cell states that are clonally expanded [82]. These $\mathrm{CD} 4^{+} \mathrm{T}$ cells can kill autologous tumors in an MHC class II-dependent manner and are suppressed by regulatory $\mathrm{T}$ cells. A gene signature of cytotoxic $\mathrm{CD} 4^{+}$ $\mathrm{T}$ cells predicted the clinical response of patients with metastatic bladder cancer treated with anti-PD-L1.

Stoeckius et al. developed cellular indexing of transcriptomes and epitopes by sequencing (CITE-seq), a method in which oligonucleotide-labeled antibodies are used to measure the expression levels of surface proteins, which is essential for immunoprofiling [83]. Granja et al. integrated CITE-seq with single-cell ATAC-seq of leukemia cells from patients with mixed-phenotype acute leukemia and showed that CD69 activation, regulated by RUNX1, is associated with poor prognosis [84]. Mimitou et al. established expanded CRISPR-compatible CITE-seq, in which CITEseq was combined with a cell hashing method for multiplexing and double detection [85], $5^{\prime}$ capture-based cDNA library generation for clonal analysis, and a system for the direct and robust capture of sgRNAs, enabling the simultaneous analysis of RNA expression, protein expression, clonality, perturbation, and cell labeling [86].

\section{Clinical application and future perspectives}

Single-cell genomics has been utilized for delineating the molecular behavior of rare clinical samples and their relationship with patients' phenotypes [87]. Velmeshev et al. used single-nucleus RNA-seq of cortical tissue from patients with autism and found that the synaptic signaling of upper-layer excitatory neurons is affected in autism and that dysregulation of specific groups of genes in cortico-cortical projection neurons correlates with clinical severity [88]. The causality of these genes was validated by large-scale exome sequencing [89]. Mathys et al. analyzed single-nucleus transcriptomes from the prefrontal cortex of patients with varying degrees of Alzheimer's disease pathology, highlighting myelination-related genes as pathogenic, and revealed that the disease-associated changes emerge early in pathological progression and are highly cell-type-specific, whereas genes upregulated at the late phase are common across cell types and involved in the global stress response [90]. Kim et al. performed single-cell RNA-seq on skin and blood samples from a patient with refractory drug-induced hypersensitivity syndrome/drug reaction with eosinophilia and systemic symptoms and identified JAK-STAT pathway activation in memory $\mathrm{CD}^{+}$cells in which DNA from human herpesvirus $6 \mathrm{~b}$ is detected [91]. They also demonstrated that tofacitinib, a JAK-STAT pathway inhibitor, suppresses T-cell proliferation. Reyes et al. conducted single-cell RNA-seq to profile peripheral blood mononuclear cells and dendritic cells from patients with sepsis and identified a unique subset of $\mathrm{CD} 14^{+}$cells in which FOS-Jun, PU.1, and CEBP are activated to regulate immune-related gene expression [92]. Smillie et al. generated a single-cell atlas of the colonic mucosa from patients with ulcerative colitis, identified IL13RA2 ${ }^{+} \mathrm{IL}_{1} 1^{+}$inflammatory fibroblasts as being associated with resistance to anti-TNF treatment, and deployed single-cell co-expression analysis to highlight putative causal genes for inflammatory bowel disease [93]. By combining single-cell RNA sequencing with spatial transcriptomics and single-cell pathology analysis, Ji et al. defined the cellular composition and architecture of cutaneous squamous cell carcinoma and identified a tumorspecific keratinocyte population that localized to a fibrovascular niche [94]. They also used in vivo CRISPR screens to identify essential roles for specific tumor subpopulationenriched gene networks in tumorigenesis.

Further utilization of single-cell genomics analysis using clinical samples to dissect pathology is advancing, but the importance of bulk sample analysis, which does not require specialized equipment and rigorous cell isolation and enables the processing of many samples, will be maintained. There are algorithms to characterize cell-type composition across subjects from bulk RNA-seq data using single-cell RNA-seq profiles as references [95-97]. Wang et al. developed multi-subject single-cell deconvolution to characterize cell-type composition from bulk RNA-seq data of the kidney and revealed that the proportion of distal convoluted tubule cells increases with disease progression [95]. By using single-cell RNA-seq profiles to deconvolute expression data from the Genotype-Tissue Expression (GTEx) project, Donovan et al. discovered cell-typespecific expression quantitative trait loci [97].

The batch effect, which is caused by differences in the conditions of sample collection and preservation, the efficiency of cDNA library synthesis, or the number of sequencing reads, should be reduced as much as possible to integrate multiple datasets for large-scale single-cell genomics analysis. Data integration algorithms such as LIGER [98], Seurat v3 [99], Scanorama [100], and Harmony [101] have been developed and widely used to integrate not only multiple single-cell RNA-seq datasets but also single-cell RNA-seq datasets and single-cell epigenomic datasets (e.g., ATAC and DNA methylation) or spatial omics datasets.

In the near future, the number of studies integrating single-cell genomics with deep phenotyping [102] or assessing/predicting drug responses with single-cell 
genomics will increase [103-105]. After confirming the conservation of the myeloid subsets in human and mouse colorectal cancer, Zhang et al. used single-cell RNA-seq to show that anti-CSF1R treatment preferentially depletes macrophages with an inflammatory signature, but spares macrophage populations that express pro-angiogenic/ tumorigenic genes, and that CD40 agonist treatment preferentially activates a specific dendritic cell population and expands Th1-like and $\mathrm{CD}^{+}$memory $\mathrm{T}$ cells [106]. Srivatsan et al. used a sample labeling (hashing) strategy that relies on labeling nuclei with unmodified single strand DNA oligonucleotides to develop single-cell combinatorial indexing and applied it to high-throughput screens on three cancer cell lines [107]. They profiled 649,340 single-cell transcriptomes across 4608 independent samples in one experiment and identified histone deacetylase inhibitors as inducers of an acetyl-CoA-deprived state.

Histone modifications and alternative splicing are critical for transcriptional regulation; therefore, the development of methods to analyze them quantitatively at single-cell resolution will lead to a deeper understanding of the molecular mechanisms underlying transcriptional regulation in clinical samples. Henikoff and colleagues developed cleavage under targets and release using nuclease, which is an epigenomic profiling strategy in which antibody-targeted controlled cleavage by micrococcal nuclease releases specific protein-DNA complexes into the supernatant for sequencing, and reduced the number of cells required for epigenomic analysis [108, 109]. Hainer et al. applied this method to nuclear samples and performed genome-wide analyses of histone modifications and transcription factor binding at single-cell resolution [110]. By using a protein A-Tn5 transposase fusion protein, Henikoff and colleagues developed cleavage under targets and tagmentation, in which antibody-targeted controlled tethering of transposase is used to generate fragment libraries, and enabled epigenomic profiling of single cells [111]. Recently, Hagemann-Jensen et al. developed Smart-seq3, which combines full-length transcriptome coverage with a $5^{\prime}$ unique molecular identifier RNA counting strategy, enabling the reconstruction of thousands of RNA molecules per cell in silico [112]. Smartseq3 has greatly increased sensitivity compared to Smartseq 2 and reconstructed isoform-specific RNA molecules, providing the opportunity to investigate isoform-level RNA quantification at the single-cell level.

\section{Conclusion}

As I have summarized in this review, single-cell genomics has been combined with a variety of technologies and has uncovered hidden molecular mechanisms in several biological phenomena, including development, physiology, and pathophysiology. In the near future, the integration of multidimensional datasets obtained through single-cell genomics approaches will have a major impact on biological research and clinical pathology. I fully expect that the implementation and expansion of single-cell genomics will lead to vast improvements in the diagnosis, stratification, and treatment of patients worldwide.

Acknowledgements This work was supported by a Grant-in-Aid for Scientific Research (B) and grants from the Japan Foundation for Applied Enzymology, SENSHIN Medical Research Foundation, KANAE Foundation for the Promotion of Medical Science, MSD Life Science Foundation, Tokyo Biomedical Research Foundation, Astellas Foundation for Research on Metabolic Disorders, Novartis Foundation (Japan) for the Promotion of Science, the Japanese Circulation Society, Takeda Science Foundation, and AMED (JP20gm0810013, JP20ek0109440, JP20ek0109487, JP20ek0109406, JP20km0405209, JP20bm0704026, JP20gm6210010, JP20ek0210141, JP19bm0804010).

\section{Compliance with ethical standards}

Conflict of interest The author declare no conflict of interets.

Publisher's note Springer Nature remains neutral with regard to jurisdictional claims in published maps and institutional affiliations.

Open Access This article is licensed under a Creative Commons Attribution 4.0 International License, which permits use, sharing, adaptation, distribution and reproduction in any medium or format, as long as you give appropriate credit to the original author(s) and the source, provide a link to the Creative Commons license, and indicate if changes were made. The images or other third party material in this article are included in the article's Creative Commons license, unless indicated otherwise in a credit line to the material. If material is not included in the article's Creative Commons license and your intended use is not permitted by statutory regulation or exceeds the permitted use, you will need to obtain permission directly from the copyright holder. To view a copy of this license, visit http://creativecommons. org/licenses/by/4.0/.

\section{References}

1. See K, Tan WLW, Lim EH, et al. Single cardiomyocyte nuclear transcriptomes reveal a lincRNA-regulated de-differentiation and cell cycle stress-response in vivo. Nat Commun. 2017;8:225. https://doi.org/10.1038/s41467-017-00319-8.

2. Picelli S, Björklund ÅK, Faridani OR, Sagasser S, Winberg G, Sandberg R. Smart-seq2 for sensitive full-length transcriptome profiling in single cells. Nat Methods. 2013;10:1096-8. https:// doi.org/10.1038/nmeth.2639.

3. Picelli S, Faridani OR, Björklund AK, Winberg G, Sagasser S, Sandberg R. Full-length RNA-seq from single cells using Smartseq2. Nat Protoc. 2014;9:171-81. https://doi.org/10.1038/nprot. 2014.006.

4. Nomura S, Satoh M, Fujita T, et al. Cardiomyocyte gene programs encoding morphological and functional signatures in cardiac hypertrophy and failure. Nat Commun. 2018;9:4435. https://doi.org/10.1038/s41467-018-06639-7.

5. Langfelder P, Horvath S. WGCNA: an R package for weighted correlation network analysis. BMC Bioinforma. 2008;9:559. https://doi.org/10.1186/1471-2105-9-559. 
6. Qiu X, Mao Q, Tang Y, et al. Reversed graph embedding resolves complex single-cell trajectories. Nat Methods. 2017;14:979-82. https://doi.org/10.1038/nmeth.4402.

7. Wang F, Flanagan J, Su N, et al. RNAscope: a novel in situ RNA analysis platform for formalin-fixed, paraffin-embedded tissues. J Mol Diagn 2012;14:22-9. https://doi.org/10.1016/j.jmoldx.2011. 08.002 .

8. Satoh M, Nomura S, Harada M, et al. High-throughput singlemolecule RNA imaging analysis reveals heterogeneous responses of cardiomyocytes to hemodynamic overload. J Mol Cell Cardiol. 2019;128:77-89. https://doi.org/10.1016/j.yjmcc.2018.12.018.

9. Ko T, Fujita K, Nomura S, et al. Quantification of DNA damage in heart tissue as a novel prediction tool for therapeutic prognosis of patients with dilated cardiomyopathy. JACC Basic Transl Sci. 2019;4:670-80. https://doi.org/10.1016/j.jacbts.2019.05.010.

10. Martini E, Kunderfranco P, Peano C, et al. Single-cell sequencing of mouse heart immune infiltrate in pressure overload-driven heart failure reveals extent of immune activation. Circulation. 2019;140:2089-107. https://doi.org/10.1161/ CIRCULATIONAHA.119.041694.

11. Tucker NR, Chaffin M, Fleming SJ, et al. Transcriptional and Cellular Diversity of the Human Heart. Circulation. 2020. https:// doi.org/10.1161/CIRCULATIONAHA.119.045401.

12. Wang L, Yu P, Zhou B, et al. Single-cell reconstruction of the adult human heart during heart failure and recovery reveals the cellular landscape underlying cardiac function. Nat Cell Biol. 2020;22:108-19. https://doi.org/10.1038/s41556-019-0446-7.

13. Cohen M, Giladi A, Gorki AD, et al. Lung single-cell signaling interaction map reveals basophil role in macrophage imprinting. Cell. 2018;175:1031-44.e18. https://doi.org/10.1016/j.cell.2018. 09.009 .

14. Vento-Tormo R, Efremova M, Botting RA, et al. Single-cell reconstruction of the early maternal-fetal interface in humans. Nature. 2018;563:347-53. https://doi.org/10.1038/s41586-0180698-6.

15. Efremova M, Vento-Tormo M, Teichmann SA, Vento-Tormo R. CellPhoneDB: inferring cell-cell communication from combined expression of multi-subunit ligand-receptor complexes. Nat Protoc. 2020;15:1484-506. https://doi.org/10.1038/s41596-020-0292-x.

16. Giladi A, Cohen M, Medaglia C, et al. Dissecting cellular crosstalk by sequencing physically interacting cells. Nat Biotechnol. 2020;38:629-37. https://doi.org/10.1038/s41587-0200442-2.

17. Ma S, Sun S, Geng L, et al. Caloric restriction reprograms the single-cell transcriptional landscape of rattus norvegicus aging. Cell. 2020;180:984-1001.e22. https://doi.org/10.1016/j.cell. 2020.02.008.

18. Aibar S, González-Blas CB, Moerman T, et al. SCENIC: singlecell regulatory network inference and clustering. Nat Methods. 2017;14:1083-6. https://doi.org/10.1038/nmeth.4463.

19. Femino AM, Fay FS, Fogarty K, Singer RH. Visualization of single RNA transcripts in situ. Science. 1998;280:585-90. https://doi.org/10.1126/science.280.5363.585.

20. Eng CL, Lawson M, Zhu Q, et al. Transcriptome-scale superresolved imaging in tissues by RNA seqFISH. Nature. 2019;568:235-9. https://doi.org/10.1038/s41586-019-1049-y.

21. Su JH, Zheng P, Kinrot SS, Bintu B, Zhuang X. Genome-scale imaging of the $3 \mathrm{D}$ organization and transcriptional activity of chromatin. Cell. 2020;S0092-8674:30940-5. https://doi.org/10. 1016/j.cell.2020.07.032.

22. Mayr U, Serra D, Liberali P. Exploring single cells in space and time during tissue development, homeostasis and regeneration. Development. 2019;146:dev176727. https://doi.org/10.1242/dev. 176727.

23. Goltsev Y, Samusik N, Kennedy-Darling J, et al. Deep profiling of mouse splenic architecture with CODEX multiplexed imaging. Cell. 2018;174:968-81.e15. https://doi.org/10.1016/j. cell.2018.07.010

24. Schürch CM, Bhate SS, Barlow GL, et al. Coordinated cellular neighborhoods orchestrate antitumoral immunity at the colorectal cancer invasive front. Cell. 2020;S0092-8674:30870-9. https:// doi.org/10.1016/j.cell.2020.07.005.

25. Jackson HW, Fischer JR, Zanotelli VRT, et al. The single-cell pathology landscape of breast cancer. Nature. 2020;578:615-20. https://doi.org/10.1038/s41586-019-1876-x.

26. Rodriques SG, Stickels RR, Goeva A, et al. Slide-seq: a scalable technology for measuring genome-wide expression at high spatial resolution. Science. 2019;363:1463-7. https://doi.org/10. 1126/science.aaw1219.

27. Ståhl PL, Salmén F, Vickovic S, et al. Visualization and analysis of gene expression in tissue sections by spatial transcriptomics. Science. 2016;353:78-82. https://doi.org/10.1126/science.aa f2403.

28. Asp M, Giacomello S, Larsson L, et al. A spatiotemporal organwide gene expression and cell atlas of the developing human heart. Cell. 2019;179:1647-60.e19. https://doi.org/10.1016/j.cell. 2019.11.025.

29. Ueda HR, Ertürk A, Chung K, et al. Tissue clearing and its applications in neuroscience. Nat Rev Neurosci. 2020;21:61-79. https://doi.org/10.1038/s41583-019-0250-1.

30. Li W, Germain RN, Gerner MY. High-dimensional cell-level analysis of tissues with Ce3D multiplex volume imaging. Nat Protoc. 2019;14:1708-33. https://doi.org/10.1038/s41596-0190156-4.

31. Trapnell C, Cacchiarelli D, Grimsby J, et al. The dynamics and regulators of cell fate decisions are revealed by pseudotemporal ordering of single cells. Nat Biotechnol. 2014;32:381-6. https:// doi.org/10.1038/nbt.2859.

32. Cao J, Spielmann M, Qiu X, et al. The single-cell transcriptional landscape of mammalian organogenesis. Nature. 2019;566:496-502. https://doi.org/10.1038/s41586-019-0969-х.

33. Packer JS, Zhu Q, Huynh C, et al. A lineage-resolved molecular atlas of C. elegans embryogenesis at single-cell resolution. Science. 2019;365:eaax1971. https://doi.org/10.1126/science.aa x1971.

34. McInnes, L, Healy, J \& Melville, J UMAP: Uniform manifold approximation and projection for dimension reduction. Stat. Mach. Learn. arXiv preprint arXiv:1802.03426. 2018.

35. Dorrity MW, Saunders LM, Queitsch C, Fields S, Trapnell C. Dimensionality reduction by UMAP to visualize physical and genetic interactions. Nat Commun. 2020;11:1537. https://doi. org/10.1038/s41467-020-15351-4.

36. La Manno G, Soldatov R, Zeisel A, et al. RNA velocity of single cells. Nature 2018;560:494-8. https://doi.org/10.1038/s41586018-0414-6.

37. Wagner DE, Klein AM. Lineage tracing meets single-cell omics: opportunities and challenges. Nat Rev Genet. 2020;10. https:// doi.org/10.1038/s41576-020-0223-2.

38. Biddy BA, Kong W, Kamimoto K, et al. Single-cell mapping of lineage and identity in direct reprogramming. Nature. 2018;564:219-24. https://doi.org/10.1038/s41586-018-0744-4.

39. Weinreb C, Rodriguez-Fraticelli A, Camargo FD, Klein AM. Lineage tracing on transcriptional landscapes links state to fate during differentiation. Science. 2020;367:eaaw3381. https://doi. org/10.1126/science.aaw3381.

40. Pei W, Feyerabend TB, Rössler J, et al. Polylox barcoding reveals haematopoietic stem cell fates realized in vivo. Nature. 2017;548:456-60. https://doi.org/10.1038/nature23653.

41. Pei W, Wang X, Rössler J, Feyerabend TB, Höfer T, Rodewald HR. Using Cre-recombinase-driven Polylox barcoding for in vivo fate mapping in mice. Nat Protoc. 2019;14:1820-40. https://doi.org/10.1038/s41596-019-0163-5. 
42. Pei W, Shang F, Wang X, et al. Resolving fate and transcriptome of hematopoietic stem cell clones. bioRxiv. 2020. https://doi.org/ 10.1101/2020.03.25.008433.

43. Chan MM, Smith ZD, Grosswendt S, et al. Molecular recording of mammalian embryogenesis. Nature. 2019;570:77-82. https:// doi.org/10.1038/s41586-019-1184-5.

44. Kalhor R, Kalhor K, Mejia L, et al. Developmental barcoding of whole mouse via homing CRISPR. Science. 2018;361:eaat9804. https://doi.org/10.1126/science.aat9804.

45. Alemany A, Florescu M, Baron CS, Peterson-Maduro J, van Oudenaarden A. Whole-organism clone tracing using single-cell sequencing. Nature. 2018;556:108-12. https://doi.org/10.1038/ nature25969.

46. Bowling S, Sritharan D, Osorio FG, et al. An engineered CRISPR-Cas9 mouse line for simultaneous readout of lineage histories and gene expression profiles in single cells. Cell. 2020; S0092-8674:30554-7. https://doi.org/10.1016/j.cell.2020.04.048.

47. Baron CS, van Oudenaarden A. Unravelling cellular relationships during development and regeneration using genetic lineage tracing. Nat Rev Mol Cell Biol. 2019;20:753-65. https://doi.org/ 10.1038/s41580-019-0186-3.

48. Frieda KL, Linton JM, Hormoz S, et al. Synthetic recording and in situ readout of lineage information in single cells. Nature. 2017;541:107-11. https://doi.org/10.1038/nature20777.

49. Macaulay IC, Haerty W, Kumar P, et al. G\&T-seq: parallel sequencing of single-cell genomes and transcriptomes. Nat Methods. 2015;12:519-22. https://doi.org/10.1038/nmeth.3370.

50. Macaulay IC, Teng MJ, Haerty W, Kumar P, Ponting CP, Voet T. Separation and parallel sequencing of the genomes and transcriptomes of single cells using G\&T-seq. Nat Protoc. 2016;11:2081-103. https://doi.org/10.1038/nprot.2016.138.

51. Dey SS, Kester L, Spanjaard B, Bienko M, van Oudenaarden A. Integrated genome and transcriptome sequencing of the same cell. Nat Biotechnol. 2015;33:285-9. https://doi.org/10.1038/nbt. 3129.

52. Liu F, Zhang Y, Zhang L, et al. Systematic comparative analysis of single-nucleotide variant detection methods from single-cell RNA sequencing data. Genome Biol. 2019;20:242. https://doi. org/10.1186/s13059-019-1863-4.

53. Enge M, Arda HE, Mignardi M, et al. Single-cell analysis of human pancreas reveals transcriptional signatures of aging and somatic mutation patterns. Cell. 2017;171:321-30.e14. https:// doi.org/10.1016/j.cell.2017.09.004.

54. Nam AS, Kim KT, Chaligne R, et al. Somatic mutations and cell identity linked by genotyping of transcriptomes. Nature. 2019;571:355-60. https://doi.org/10.1038/s41586-019-1367-0.

55. Angermueller C, Clark SJ, Lee HJ, et al. Parallel single-cell sequencing links transcriptional and epigenetic heterogeneity. Nat Methods. 2016;13:229-32. https://doi.org/10.1038/nmeth.3728.

56. Pott S. Simultaneous measurement of chromatin accessibility, DNA methylation, and nucleosome phasing in single cells. Elife. 2017;6:e23203. https://doi.org/10.7554/eLife.23203.

57. Clark SJ, Argelaguet R, Kapourani CA, et al. scNMT-seq enables joint profiling of chromatin accessibility DNA methylation and transcription in single cells. Nat Commun. 2018;9:781. https://doi.org/10.1038/s41467-018-03149-4.

58. Argelaguet R, Clark SJ, Mohammed $\mathrm{H}$, et al. Multi-omics profiling of mouse gastrulation at single-cell resolution. Nature. 2019;576:487-91. https://doi.org/10.1038/s41586-019-1825-8.

59. Liu L, Liu C, Quintero A, et al. Deconvolution of single-cell multi-omics layers reveals regulatory heterogeneity. Nat Commun. 2019;10:470. https://doi.org/10.1038/s41467-018-08205-7.

60. Zhu C, Yu M, Huang H, et al. An ultra high-throughput method for single-cell joint analysis of open chromatin and transcriptome. Nat Struct Mol Biol. 2019;26:1063-70. https://doi. org/10.1038/s41594-019-0323-x.
61. Chen S, Lake BB, Zhang K. High-throughput sequencing of the transcriptome and chromatin accessibility in the same cell. Nat Biotechnol. 2019;37:1452-7. https://doi.org/10.1038/s41587019-0290-0.

62. Rooijers K, Markodimitraki CM, Rang FJ, et al. Simultaneous quantification of protein-DNA contacts and transcriptomes in single cells. Nat Biotechnol. 2019;37:766-72. https://doi.org/10. 1038/s41587-019-0150-y.

63. Markodimitraki CM, Rang FJ, Rooijers K, et al. Simultaneous quantification of protein-DNA interactions and transcriptomes in single cells with scDam\&T-seq. Nat Protoc. 2020;15:1922-53. https://doi.org/10.1038/s41596-020-0314-8.

64. Mateo LJ, Murphy SE, Hafner A, Cinquini IS, Walker CA, Boettiger AN. Visualizing DNA folding and RNA in embryos at single-cell resolution. Nature. 2019;568:49-54. https://doi.org/ 10.1038/s41586-019-1035-4.

65. Katzenelenbogen Y, Sheban F, Yalin A, et al. Coupled scRNASeq and intracellular protein activity reveal an immunosuppressive role of TREM2 in cancer. Cell. 2020;182:872-85.e19. https://doi.org/10.1016/j.cell.2020.06.032.

66. Dixit A, Parnas O, Li B, et al. Perturb-seq: dissecting molecular circuits with scalable single-cell rna profiling of pooled genetic screens. Cell. 2016;167:1853-66.e17. https://doi.org/10.1016/j. cell.2016.11.038.

67. Adamson B, Norman TM, Jost M, et al. A multiplexed singlecell CRISPR screening platform enables systematic dissection of the unfolded protein response. Cell. 2016;167:1867-82.e21. https://doi.org/10.1016/j.cell.2016.11.048.

68. Jaitin DA, Weiner A, Yofe I, et al. Dissecting immune circuits by linking CRISPR-pooled screens with single-cell RNA-seq. Cell. 2016;167:1883-96.e15. https://doi.org/10.1016/j.cell.2016.11.039.

69. Datlinger P, Rendeiro AF, Schmidl C, et al. Pooled CRISPR screening with single-cell transcriptome readout. Nat Methods. 2017;14:297-301. https://doi.org/10.1038/nmeth.4177.

70. Hill AJ, McFaline-Figueroa JL, Starita LM, et al. On the design of CRISPR-based single-cell molecular screens. Nat Methods. 2018;15:271-4. https://doi.org/10.1038/nmeth.4604.

71. Norman TM, Horlbeck MA, Replogle JM, et al. Exploring genetic interaction manifolds constructed from rich single-cell phenotypes. Science. 2019;365:786-93. https://doi.org/10.1126/ science.aax4438.

72. Jost M, Santos DA, Saunders RA, et al. Titrating gene expression using libraries of systematically attenuated CRISPR guide RNAs. Nat Biotechnol. 2020;38:355-64. https://doi.org/10. 1038/s41587-019-0387-5.

73. Replogle JM, Norman TM, Xu A, et al. Combinatorial singlecell CRISPR screens by direct guide RNA capture and targeted sequencing. Nat Biotechnol. 2020. https://doi.org/10.1038/ s41587-020-0470-y.

74. Rubin AJ, Parker KR, Satpathy AT, et al. Coupled single-cell CRISPR screening and epigenomic profiling reveals causal gene regulatory networks. Cell. 2019;176:361-76.e17. https://doi.org/ 10.1016/j.cell.2018.11.022.

75. Gasperini M, Hill AJ, McFaline-Figueroa JL, et al. A genomewide framework for mapping gene regulation via cellular genetic screens. Cell. 2019;176:377-90.e19. https://doi.org/10.1016/j. cell.2018.11.029.

76. Wroblewska A, Dhainaut M, Ben-Zvi B, et al. Protein barcodes enable high-dimensional single-cell CRISPR screens. Cell. 2018;175:1141-55.e16. https://doi.org/10.1016/j.cell.2018.09. 022.

77. Feldman D, Singh A, Schmid-Burgk JL, et al. Optical pooled screens in human cells. Cell. 2019;179:787-99.e17. https://doi. org/10.1016/j.cell.2019.09.016.

78. Park JE, Botting RA, Domínguez Conde C, et al. A cell atlas of human thymic development defines $\mathrm{T}$ cell repertoire formation. 
Science. 2020;367:eaay3224. https://doi.org/10.1126/science.aa y3224.

79. Wu TD, Madireddi S, de Almeida PE, et al. Peripheral T cell expansion predicts tumour infiltration and clinical response. Nature. 2020;579:274-8. https://doi.org/10.1038/s41586-020-2056-8.

80. Gate D, Saligrama N, Leventhal O, et al. Clonally expanded CD8 $\mathrm{T}$ cells patrol the cerebrospinal fluid in Alzheimer's disease. Nature. 2020;577:399-404. https://doi.org/10.1038/s41586-0191895-7.

81. Glanville J, Huang H, Nau A, et al. Identifying specificity groups in the T cell receptor repertoire. Nature. 2017;547:94-8. https:// doi.org/10.1038/nature22976.

82. Oh DY, Kwek SS, Raju SS, et al. Intratumoral CD4+ T cells mediate anti-tumor cytotoxicity in human bladder cancer. Cell. 2020;S0092-8674:30612-7. https://doi.org/10.1016/j.cell.2020. 05.017.

83. Stoeckius M, Hafemeister C, Stephenson W, et al. Simultaneous epitope and transcriptome measurement in single cells. Nat Methods. 2017;14:865-8. https://doi.org/10.1038/nmeth.4380.

84. Granja JM, Klemm S, McGinnis LM, et al. Single-cell multiomic analysis identifies regulatory programs in mixed-phenotype acute leukemia. Nat Biotechnol. 2019;37:1458-65. https://doi.org/10. 1038/s41587-019-0332-7.

85. Stoeckius M, Zheng S, Houck-Loomis B, et al. Cell Hashing with barcoded antibodies enables multiplexing and doublet detection for single cell genomics. Genome Biol. 2018;19:224. https://doi.org/10.1186/s13059-018-1603-1.

86. Mimitou EP, Cheng A, Montalbano A, et al. Multiplexed detection of proteins, transcriptomes, clonotypes and CRISPR perturbations in single cells. Nat Methods. 2019;16:409-12. https://doi.org/10.1038/s41592-019-0392-0.

87. Lim B, Lin Y, Navin N. Advancing cancer research and medicine with single-cell genomics. Cancer Cell. 2020;37:456-70. https://doi.org/10.1016/j.ccell.2020.03.008.

88. Velmeshev D, Schirmer L, Jung D, et al. Single-cell genomics identifies cell type-specific molecular changes in autism. Science. 2019;364:685-9. https://doi.org/10.1126/science.aav8130.

89. Satterstrom FK, Kosmicki JA, Wang J, et al. Large-scale exome sequencing study implicates both developmental and functional changes in the neurobiology of autism. Cell. 2020;180:568-84. e23. https://doi.org/10.1016/j.cell.2019.12.036.

90. Mathys H, Davila-Velderrain J, Peng Z, et al. Single-cell transcriptomic analysis of Alzheimer's disease. Nature. 2019;570:332-7. https://doi.org/10.1038/s41586-019-1195-2.

91. Kim D, Kobayashi T, Voisin B, et al. Targeted therapy guided by single-cell transcriptomic analysis in drug-induced hypersensitivity syndrome: a case report. Nat Med. 2020;26:236-43. https://doi.org/10.1038/s41591-019-0733-7.

92. Reyes M, Filbin MR, Bhattacharyya RP, et al. An immune-cell signature of bacterial sepsis. Nat Med. 2020;26:333-40. https:// doi.org/10.1038/s41591-020-0752-4.

93. Smillie CS, Biton M, Ordovas-Montanes J, et al. Intra- and intercellular rewiring of the human colon during ulcerative colitis. Cell. 2019;178:714-30.e22. https://doi.org/10.1016/j.cell.2019.06.029.

94. Ji AL, Rubin AJ, Thrane K, et al. Multimodal analysis of composition and spatial architecture in human squamous cell carcinoma. Cell. 2020;182:497-514.e22. https://doi.org/10.1016/j. cell.2020.05.039.

95. Wang X, Park J, Susztak K, Zhang NR, Li M. Bulk tissue cell type deconvolution with multi-subject single-cell expression reference. Nat Commun. 2019;10:380. https://doi.org/10.1038/ s41467-018-08023-x.
96. Frishberg A, Peshes-Yaloz N, Cohn O, et al. Cell composition analysis of bulk genomics using single-cell data. Nat Methods. 2019;16:327-32. https://doi.org/10.1038/s41592-019-0355-5.

97. Donovan MKR, D'Antonio-Chronowska A, D'Antonio M, Frazer KA. Cellular deconvolution of GTEx tissues powers discovery of disease and cell-type associated regulatory variants. Nat Commun 2020;11:955. https://doi.org/10.1038/s41467-02014561-0.

98. Welch JD, Kozareva V, Ferreira A, Vanderburg C, Martin C, Macosko EZ. Single-cell multi-omic integration compares and contrasts features of brain cell identity. Cell. 2019;177:1873-87. e17. https://doi.org/10.1016/j.cell.2019.05.006.

99. Stuart T, Butler A, Hoffman P, et al. Comprehensive integration of single-cell data. Cell. 2019;177:1888-902.e21. https://doi.org/ 10.1016/j.cell.2019.05.031.

100. Hie B, Bryson B, Berger B. Efficient integration of heterogeneous single-cell transcriptomes using Scanorama. Nat Biotechnol. 2019;37:685-91. https://doi.org/10.1038/s41587-019-0113-3.

101. Korsunsky I, Millard N, Fan J, et al. Fast, sensitive and accurate integration of single-cell data with Harmony. Nat Methods. 2019;16:1289-96. https://doi.org/10.1038/s41592-019-0619-0.

102. Bycroft C, Freeman C, Petkova D, Band G, Elliott LT, Sharp K, et al. The UK Biobank resource with deep phenotyping and genomic data. Nature. 2018;562:203-9.

103. Yofe I, Dahan R, Amit I. Single-cell genomic approaches for developing the next generation of immunotherapies. Nat Med. 2020;26:171-7.

104. Efremova M, Vento-Tormo R, Park JE, Teichmann SA, James KR. Immunology in the era of single-cell technologies. Annu Rev Immunol. 2020. https://doi.org/10.1146/annurev-immunol090419-020340.

105. Rozenblatt-Rosen O, Regev A, Oberdoerffer P, et al. The human tumor atlas network: charting tumor transitions across space and time at single-cell resolution. Cell. 2020;181:236-49. https://doi. org/10.1016/j.cell.2020.03.053.

106. Zhang L, Li Z, Skrzypczynska KM, et al. Single-cell analyses inform mechanisms of myeloid-targeted therapies in colon cancer. Cell. 2020;181:442-59.e29. https://doi.org/10.1016/j.cell. 2020.03.048.

107. Srivatsan SR, McFaline-Figueroa JL, Ramani V, et al. Massively multiplex chemical transcriptomics at single-cell resolution. Science. 2020;367:45-51. https://doi.org/10.1126/science.aa x6234.

108. Skene PJ, Henikoff S. An efficient targeted nuclease strategy for high-resolution mapping of DNA binding sites. Elife. 2017;6: e21856. https://doi.org/10.7554/eLife.21856.

109. Skene PJ, Henikoff JG, Henikoff S. Targeted in situ genomewide profiling with high efficiency for low cell numbers. Nat Protoc. 2018;13:1006-19. https://doi.org/10.1038/nprot.2018. 015.

110. Hainer SJ, Bošković A, McCannell KN, Rando OJ, Fazzio TG. Profiling of pluripotency factors in single cells and early embryos. Cell. 2019;177:1319-29.e11. https://doi.org/10.1016/j. cell.2019.03.014.

111. Kaya-Okur HS, Wu SJ, Codomo CA, et al. CUT\&Tag for efficient epigenomic profiling of small samples and single cells. Nat Commun. 2019;10:1930. https://doi.org/10.1038/s41467-01909982-5.

112. Hagemann-Jensen $M$, Ziegenhain $C$, Chen $P$, et al. Singlecell RNA counting at allele and isoform resolution using Smartseq3. Nat Biotechnol. 2020;38:708-14. https://doi.org/10.1038/ s41587-020-0497-0. 